\title{
XXII. Correlated averages
}

\section{Professor F. Y. Edgeworth M.A. D.C.L.}

To cite this article: Professor F. Y. Edgeworth M.A. D.C.L. (1892) XXII. Correlated averages, Philosophical Magazine Series 5, 34:207, 190-204, DOI: 10.1080/14786449208620307

To link to this article: http://dx.doi.org/10.1080/14786449208620307

$$
\text { 曲 Published online: } 07 \text { May } 2010 .
$$

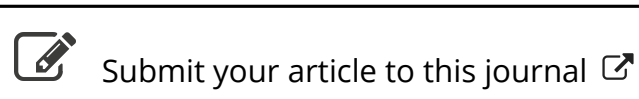
Lll Article views: 6

Q View related articles $₫$

7 Citing articles: 16 View citing articles 


\section{[ 190 ]}

XXII. Correlated Averages.

By Professor F. Y. EDGeworte, M.A., D.C.L.*

THE "correlation" $\dagger$ between the members of a system 1 such as the limbs or other measurable attributes of an organism may in general be expressed by the formula

$$
\Pi=\mathrm{J} e^{-\mathrm{R}} d x_{1}, d x_{2}, d x_{3}, \& c . ;
$$

where

$$
\begin{aligned}
\mathbf{R} & =p_{1}\left(\mathbf{x}_{1}-x_{1}\right)^{2}+p_{2}\left(\mathbf{x}_{2}-x_{2}\right)^{2}+\& c ., \\
& +2 q_{12}\left(\mathbf{x}_{1}-x_{1}\right)\left(\mathbf{x}_{2}-x_{2}\right)+2 q_{13}\left(\mathbf{x}_{1}-x_{1}\right)\left(\mathbf{x}_{3}-x_{3}\right)+\& c . ;
\end{aligned}
$$

$\mathbf{x}_{1}, \mathbf{x}_{2}, \mathbf{x}_{3}$ \&c. are the average values of the respective organs; $x_{1}, x_{2}$, \&c. are particular values of the same; $p_{1}, p_{2} \ldots q_{12}, q_{13}$ are constants to be obtained from observation; $J$ is a constant deduced from the condition that the integral of $\Pi$ between extreme limits should be unity. The expression $\Pi$ represents the probability that any particular values of $x_{1}, x_{y}$, \&c. should concur. It enables us to answer the questions: What is the most probable value of one deviation $x_{r}$ corresponding to assigned values $x_{1}{ }^{\prime}, x_{2}{ }^{\prime} \& \mathrm{c}$. of the other variables? and What is the dispersion of the values of $x_{r}$ about its mean (the other variables being assigned)?

This general formula for the concurrence of particular values of several organs is deducible from the proposition, proved by theory and observation, that each organ considered by itself assumes different values according to the exponential law of error. In a subsequent paper I hope to justify this principle ; at present, assuming the propriety of the abovewritten formula, I propose to show how the constants $p_{1}, p_{2} \ldots q_{12}, q_{13} \ldots$ are calculated. This problem has been solved by Mr. Galton for the case of two variables. The happy device of measuring each deviation by the corresponding quartile taken as unit enables him to express the sought quadratic in terms of a single parameter; as thus :-

$$
\mathrm{R}=\frac{x_{1}^{2}}{1-\rho^{2}}-\frac{2 \rho x_{1} x_{2}}{1-\rho^{2}}+\frac{x_{2}^{2}}{1-\rho^{2}} ;
$$

where our $\rho$ is Mr. Galton's $r$, and the $\mathbf{x}_{1}, \mathbf{x}_{2}$ of our general formula are zero. The parameter is found by observing the

* Communicated by the Author.

+ See Galton, Proc. Roy. Soc. 1888, "Co-relations and their Measurement;" and Weldon, Proc. Roy. Soc. 1892, "Certain Correlated Variations in Crangon vulgaris." 
value of $x_{1}$, say $\xi_{1}$, which most frequently corresponds to an assigned value of $x_{2}$, say $x_{2}^{\prime}$ (or vice versấ). From the equation $\frac{d R}{d x_{1}}=0$ we have

$$
\xi_{1}=\rho x_{2}^{\prime}, \quad \rho=\xi_{1} \div x_{2}{ }^{\prime} .
$$

It should be observed that for the purpose of this calculation it is not necessary, as Mr. Galton has done, to pick out the values of $x_{1}$ corresponding to each value of $x_{2}$. It is sufficient to take the sum, or the mean, of all or some of the positive, exclusive of negative, values [or negative, exclusive of positive] and the sum or mean of the corresponding values, not exclusive of negative [or positive]; and to equate

or

$$
\mathrm{S} \xi_{1}=\rho \mathrm{S} x_{2}^{\prime}, \quad \rho=\mathrm{S} \xi_{1} \div \mathrm{S} x_{2}^{\prime} ;
$$

$$
\frac{\mathrm{S} \xi_{1}}{n}=\rho \frac{\mathrm{S} x_{2}^{\prime}}{n}, \quad \rho=\frac{\mathrm{S} \xi_{1}}{n} \div \frac{\mathrm{S} x_{2}^{\prime}}{n} ;
$$

omitting perhaps the extreme observations, with respect to which the law of error is liable to break down.

For example, let it be required to find the coefficient of correlation between the stature and left cubit of adult males, from the data utilized by Mr. Galton in Table II. of his paper on "Co-Relations" (Proc. Roy. Soc. 1888, p. 138), without the trouble of the detailed selection, the elaborate "dépouillement," which the construction of his table requires. Take the cubit as the independent variable, $x_{2}$ of the last paragraph, and write down all the deviations of the cubit from its mean value) which are above zero and short of the extremity. There are ninety-three such instances-between 18.5 and 19.5 inches-among the materials which $\mathrm{Mr}$. Galton has employed in constructing his Table II. (op. cit.). The sum of these deviations is 1762.75 inches; the sum of the ninetythree concurrent deviations of stature is 6422 inches. Fach of these has to be divided by the corresponding quartile; .56 inch in the case of the cubit, 1.75 inch in the case of the statures. Thus

$$
\rho=\frac{S \xi_{1}}{S x_{2}}=\frac{1762 \cdot 75}{\cdot 56} \div \frac{6422}{1 \cdot 75}=\cdot 8 .
$$

Which is the value for the coefficient found by Mr. Galton.

In working this example I have taken the figures from the compartments of Mr. Galton's Table II. ; for instance, reckoning that there are 55 cubit-deviations " 18.5 and under $19 \cdot 0$," 
i. e. $18 \cdot 75$ nearly, and similarly 38 cubit-deviations measuring on an average $19 \cdot 25$. But it is not necessary in general (the prevalence of the law of error being presumed) for the purpose of calculating the coefficient to work up the materials as in Mr. Galton's Table II., or Table III. It suffices to take the figures from the original observations pell-mell as they come; in fact it is better to proceed thus, as we avoid the inaccuracy involved in assuming that all the observations in each small compartment, e. g. between 18.5 inches and $19 \cdot 0$ inches, have the same measure- $18 \cdot 75$ inches. I take an example from some of Mr. Galton's unmanipulated observations which he has kindly submitted to me. The raw material consists of a table like the following, each column giving the measurement of one organ, each row the measurements on one man,-in the random order in which the visitors to the Anthropometric laboratory presented themselves. Here is a specimen :-

\section{TABLE I.}

\begin{tabular}{|c|c|c|c|c|}
\hline Statures. & & & & Left Cubits. \\
\hline $72 \cdot 4$ & . & . & . & $19 \cdot 3$ \\
\hline 71.9 & & . . & . & $18 \cdot 7$ \\
\hline $71 \cdot 5$ & . & . . & . & . $18 \cdot 8$ \\
\hline 70 & & . . & . & . 18.4 \\
\hline 66 & . & . . & $\therefore$ & . 18 \\
\hline $70 \cdot 3$ & . & . . & . & $18 \cdot 6$ \\
\hline $72 \cdot 2$ & & . . & . & $19 \cdot 6$ \\
\hline 69 & . & . . & . & $18 \cdot 4$ \\
\hline 68 & $\cdot$ & . : & . & $18 \cdot 3$ \\
\hline $64 \cdot 4$ & & . . & . & . $18 \cdot 2$ \\
\hline $65 \cdot 5$ & & . . & . & . $16 \cdot 8$ \\
\hline $66 \cdot 1$ & : &. & $\dot{.}$ & . $17 \cdot 1$ \\
\hline $71 \cdot 6$ & & . . & . & . $19 \cdot 3$ \\
\hline 68.4 & & . & . & . $18 \cdot 2$ \\
\hline 65 & & . . & . & . $17 \cdot 3$ \\
\hline $63 \cdot 7$ & & . . & . & - $16 \cdot 8$ \\
\hline $62 \cdot 3$ & & & ${ }^{\circ}$ & . 16.5 \\
\hline : & & & • & \\
\hline
\end{tabular}

(There are other columns with which we are not at present concerned.) From the right column I pick out all the figures which are above 18.05 (the mean value of the cubit) and write them down in a new column, as thus :- 


\begin{tabular}{|c|c|}
\hline \multicolumn{2}{|c|}{ TABLE II. } \\
\hline $\begin{array}{l}\text { Left Cubit } \\
\text { (above 18.05). }\end{array}$ & $\begin{array}{c}\text { Corresponding } \\
\text { Stature. }\end{array}$ \\
\hline $19 \cdot 3$ & $72 \cdot 4$ \\
\hline $18 \cdot 7$ & $71 \cdot 9$ \\
\hline $18 \cdot 8$ & $71 \cdot 5$ \\
\hline $18 \cdot 4$ & 70 \\
\hline $18 \cdot 6$ & $70 \cdot 3$ \\
\hline $19 \cdot 6$ & $72 \cdot 2$ \\
\hline $18 \cdot 4$ & 69 \\
\hline $18 \cdot 3$ & 68 \\
\hline $19 \cdot 3$ & $71 \cdot 6$ \\
\hline $18 \cdot 2$ & $68 \cdot 4$ \\
\hline $18 \cdot 1$ & $68 \cdot 3$ \\
\hline $18 \cdot 7$ & $68 \cdot 4$ \\
\hline * 18 & $70 \cdot 2$ \\
\hline $19 \cdot 4$ & $71 \cdot 1$ \\
\hline $18 \cdot 8$ & $68 \cdot 3$ \\
\hline 15$) 280 \cdot 6$ & $15) 1051 \cdot 6$ \\
\hline $18 \cdot 707$ & $70 \cdot 107$ \\
\hline
\end{tabular}

The mean deviation of the cubits, in absolute measure, is $\frac{18 \cdot 707-18 \cdot 05}{.56}$. The corresponding mean deviation of the statures is $\frac{70 \cdot 107-67 \cdot 2}{1 \cdot 75}$; assuming from $\mathrm{Mr}$. Galton's tables in Proc. Roy. Soc. 1888 that 18.05 and $67 \cdot 2$ inches are the respective mean values of the culbit and stature, $\cdot 56$ and 1.75 their respective quartiles. For the coefficient of correlation we have

mean deviation of cubit in absolute measure $\div$ corresponding mean deviation of stature

$$
=1 \cdot 17 \div 1 \cdot 66=\cdot 7 \text { nearly. }
$$

The truer value is 8 , as determined by Mr. Galton from some hundreds of observations; but we must not expect an accurate result from a few observations here instanced by way of paradigm.

In the work of this example I have purposely allowed one mistake not largely affecting the result to stand by way of warning; the 18 marked with an asterisk in the second table which, being below the mean value of the cubit, has no Phil. Mag. S. 5. Vol. 34. No. 207. Aug. 1892. O 
business in a column furnishing positive values of the deviation $x_{2}$. It is clear that, if we admitted such values indiscriminately, we should obtain an indeterminate result for $\rho$.

Of course, the more elaborate tabulation employed by Mr. Galton in the paper referred to and elsewhere is useful for the purpose of verifying that the law of error is indeed fulfilled, of testing up to what distance from the mean it holds good, and for other purposes.

It may be observed that the calculation of the coefficients of correlation is not affected if we take as the unit, not the quartile, but the same $\div 476 \ldots$, the modulus of each set of observations. In what follows it is to be understood, when the contrary is not expressed, that the variables $x_{1} x_{2}$, \&c.representing deviations of different organs or attributes from their mean value-are thus measured.

To extend Mr. Galton's method to the case of three variables $x_{1}, x_{2}, x_{3}$; first determine the coefficients of correlation for each pair $\left(x_{1} x_{2}\right),\left(x_{1} x_{3}\right),\left(x_{2} x_{3}\right)$; say, $\rho_{12}, \rho_{13}, \rho_{11}$. 'Thus the probability of any particular $x_{1}$ and $x_{2}$ concurring is $\mathrm{K} e^{-\mathrm{s}} d x_{1} d x_{2}$, where $\mathrm{K}$ is a properly taken coefficient, and

$$
\mathrm{S}=\frac{x_{1}^{2}}{1-\rho_{12}^{2}}-\frac{2 x_{1} x_{2} \rho_{12}}{1-\rho_{12}^{2}}+\frac{x_{2}^{2}}{1-\rho_{12}{ }^{2}} .
$$

(See above, p. 190, and Mr. Galton's work there referred to.) Now the expression above written must be derived from the sought expression $\mathrm{J}^{-\mathrm{R}} d x_{1} d x_{2} d x_{3}$, where

$$
\mathbf{R}=p_{1} x_{1}^{2}+p_{2} x_{2}^{2}+p_{3} x_{3}^{2}+2 q_{12} x_{1} x_{2}+2 q_{13} x_{1} x_{3}+2 q_{23} x_{2} x_{3},
$$

by integration with respect to $x_{3}$ between extreme limits $+\infty$ and $-\infty$. If we watch the process of integration, we shall find that the coefficient of $x_{1}^{2}$ (in the exponent of the integral) is

$$
\frac{p_{2} p_{3}-q_{13}^{2}}{p_{3}}
$$

the coefficient of $x_{1} x_{2}$ is

and the coefficient of $x_{2}{ }^{2}$ is

$$
2 \frac{q_{12} p_{3}-q_{13} q_{23}}{p_{3}}
$$

$$
\frac{p_{1} p_{3}-q_{23}^{2}}{p_{3}} \text {. }
$$

These coefficients are to be equated respectively to

$$
\frac{1}{1-\rho_{12}{ }^{2}}, \frac{-2 \rho_{12}}{1-\rho_{12}{ }^{2}}, \text { and } \frac{1}{1-\rho_{12}^{2}},
$$


the coefficients of $x_{1}{ }^{2}, a_{1} a_{2}$, and $x_{2}^{2}$, in the quadratic above written considered as derived directly from the Galtonian coefficient $\rho_{12}$. Employing the quadratic to determine the most probable $u_{1}$ corresponding to any assigned $x_{2}$ (cf. above, p. 19i) we have

This may be written

$$
\rho_{12}=\frac{q_{13} q_{23}-q_{12}}{p_{2} p_{3}-q_{13}{ }^{2}}
$$

$$
\rho_{12}=\frac{\mathrm{Q}_{12}}{\mathrm{P}_{1}}
$$

where $Q_{12}$ and $P_{1}$ are certain minors of the determinant $\Delta$, which forms the discriminant of $\mathbf{R}$;

$$
\Delta=\left|\begin{array}{lll}
p_{1}, & q_{12}, & q_{13} \\
q_{12}, & p_{2}, & q_{23} \\
q_{13}, & q_{23}, & p_{3}
\end{array}\right|
$$

If we carry the integration one step further, integrating $\mathrm{K} e^{-\mathrm{s}}$ with respect to $x_{2}$ between extreme limits, we shall find for the probability of any particular $x_{1}$ the expression

$$
L e^{-x_{2} \frac{\Delta}{P_{2}}} d x_{1} \text {. }
$$

But by convention the modulus of the probability-curve under which the values of $x_{1}$ range is unity (above, p. 194). Therefore $\Delta=\mathrm{P}_{2}$.

By parity of reasoning,

$$
\begin{gathered}
\Delta=\mathrm{P}_{3}=\mathrm{P}_{1} . \\
\rho_{13}=\frac{\mathrm{Q}_{13}}{\mathrm{P}_{1}}=\frac{\mathrm{Q}_{13}}{\mathrm{P}_{3}}, \\
\rho_{23}=\frac{\mathrm{Q}_{23}}{\mathrm{P}_{2}}=\frac{\mathrm{Q}_{23}}{\mathrm{P}_{3}} .
\end{gathered}
$$

Therefore

$$
\mathrm{Q}_{12}=\Delta \rho_{12} ; \quad \mathrm{Q}_{13}=\Delta \rho_{13} ; \quad \mathrm{Q}_{23}=\Delta \rho_{23} \text {. }
$$

Thus we may write the reciprocal $\Delta^{\prime}$ of the determinant $\Delta:-$

$$
\Delta^{\prime}=\mid \begin{array}{lll}
\Delta, & \Delta \rho_{12}, & \Delta \rho_{13} \\
\Delta \rho_{12}, & \Delta, & \Delta \rho_{23} \\
\Delta \rho_{13}, & \Delta \rho_{23}, & \Delta
\end{array}
$$

But, by a well-known theorem, each first minor of $\Delta^{\prime}=\Delta$ multiplied by the corresponding constituent of $\Delta$. Thus

$$
\left.\left(\dot{\Delta}^{2}-\Delta^{2} \rho_{23}{ }^{2}\right)=\Delta p_{1} ; \quad{ }_{0}{ }_{2}^{2} \rho_{23} \rho_{13}-\Delta^{2} \rho_{12}\right)=\Delta \cdot y_{12} \cdot
$$


Whence

$$
\begin{aligned}
& p_{1}=\Delta\left(1-\rho_{23}{ }^{2}\right) \\
& q_{12}=\Delta\left(\rho_{23} \rho_{13}-\rho_{12}\right) ;
\end{aligned}
$$

with corresponding values for the other coefficients.

It remains only to determine $\Delta$. This is effected by the equation $\Delta=\mathrm{P}_{1}$. Employing the values of $p_{1}, p_{2}$ \&c., $q_{12}$, \&c., which have just been found,

whence

$$
\mathrm{P}_{1}=\left|\begin{array}{ll}
\Delta\left(1-\rho_{13}^{2}\right) & \Delta\left(\rho_{12} \rho_{23}-\rho_{13}\right) \\
\Delta\left(\rho_{12} \rho_{23}-\rho_{13}\right) & \Delta\left(1-\rho_{12}{ }^{2}\right)
\end{array}\right| ;
$$

$$
\Delta=\Delta^{2}\left\{\left(1-\rho_{13}{ }^{2}\right)\left(1-\rho_{12}{ }^{2}\right)-\left(\rho_{12} \rho_{23}-\rho_{13}\right)^{2}\right\} ;
$$

and $\Delta$ is determined in terms of the $\rho$ 's, of which the numerical values are supposed to have been ascertained. The whole system of coefficients is therefore determined numerically.

Example.-Let $x_{1}, x_{2}, x_{3}$ respectively represent deviations of stature, cubit, and height of knee. The coefficients of correlation for each pair are $\rho_{12}=\cdot 8, \rho_{13}=\cdot 9, \rho_{23}=\cdot 8$; as ascertained by Mr. Galton (Proc. Roy. Soc. 1888, Co-relations, Table V.). To find the coefficients of the expression

$$
p_{1} x_{1}^{2}+p_{2} x_{2}^{2}+p_{3} x_{3}^{2}+2 q_{12} x_{1} x_{2}+2 q_{13} x_{1} x_{3}+2 q_{23} x_{2} x,
$$

which is the exponent of the expression for the probability that any particular values of $x_{1}, x_{2}, x_{3}$ should concur; or, in other words, the equation of the ellipsoid of equal probability (the final constant being omitted).

Here for the reciprocal of the discriminant $\Delta$ we have

Whence

$$
\Delta^{\prime}=\left|\begin{array}{ccc}
\Delta, & \Delta \cdot 8, & \Delta \cdot 9 \\
\Delta \cdot 8, & \Delta, & \Delta \cdot 8 \\
\Delta \cdot 9, & \Delta \cdot 8, & \Delta
\end{array}\right| .
$$

$$
\begin{aligned}
& p_{1}=\Delta\left|\begin{array}{cc}
1, & \cdot 8 \\
8, & 1
\end{array}\right|=\Delta(1-\cdot 64)=\Delta \cdot 36, \\
& q_{12}=\Delta\left|\begin{array}{cc}
\cdot 8, & \cdot 8 \\
1, & \cdot 9
\end{array}\right|=\Delta(\cdot 72-\cdot 8)=-\Delta \cdot 08 .
\end{aligned}
$$

By parity $p_{2}=\Delta \cdot 19, p_{3}=\Delta \cdot 36, q_{13}=-\Delta \cdot 26, q_{23}=-\Delta \cdot 08$.

$$
\text { Thus } \Delta=\left|\begin{array}{rr}
\Delta \cdot 36, & -\Delta \cdot 08,-\Delta \cdot 26 \\
-\Delta \cdot 08, & \Delta \cdot 19,-\Delta \cdot 08 \\
-\Delta \cdot 26, & -\Delta \cdot 08, \\
-\Delta \cdot 36
\end{array}\right| .
$$


And to determine $\Delta$ we have

$$
\Delta=\Delta^{2}\left|\begin{array}{rr}
\cdot 19, & -\cdot 08 \\
-\cdot 08, & \cdot 36
\end{array}\right|=\Delta^{2}\left(\cdot 36 \times \cdot 19-\cdot 08^{2}\right)
$$

$=\Delta^{2} .0620$ (a result which may be verified by observing that the other two principal minors afford, as they ought, the same equation for $\Delta$ ). Whence $\Delta=16 \cdot 129$. Substituting this value in the values of the coefficients above found, we bave for the sought expression

$$
\begin{aligned}
5 \cdot 806 x_{1}{ }^{2}+3 \cdot 064 x_{2}{ }^{2}+5 \cdot 806 x_{3}{ }^{2}-2 \times 1 \cdot 290 x_{1} x_{2} \\
-2 \times 4 \cdot 194 x_{1} x_{3}-2 \times 1 \cdot 290 x_{2} x_{3} .
\end{aligned}
$$

Thus we see that the dispersion of $x_{1}$ corresponding to assigned values of $x_{2}, x_{3}$ has for modulus $\frac{1}{\sqrt{5 \cdot 806}}$. The most probable deviation of one organ, e.g. the cubit, corresponding to assigned deviations of the two other organs is found by differentiating with respect to $x_{2}$ the expression above written and equating to zero. Thus, if $x_{1}^{\prime}, x_{3}{ }^{\prime}$ be the assigned deviations of stature and height of knee, and $\xi_{2}$ the most probable corresponding deviation of cubit, $\ddot{3} \cdot 064 \xi_{2}=1 \cdot 290 x_{1}^{\prime}$

$$
+1 \cdot 290 x_{3}^{\prime} . \quad \xi_{2}=\frac{1 \cdot 290\left(u_{1}^{\prime}+x_{3}^{\prime}\right)}{3 \cdot 064}=\cdot 42\left(x_{1}^{\prime}+x_{3}^{\prime}\right) .
$$

I have verified this deduction by actually observing the value of cubit-deviation which on an average corresponds to assigned values of the other deviations. For this purpose I employ a table such as Table I. at p. 192, with an additional column for height of knee. This material, consisting of unmanipulated observations on the stature, cubit, and knee-height of three hundred men, was kindly furnished to me by Mr. Galton. Out of these three hundred triplets I pick out ninety-six which have the stature and knee-height above the respective means of those organs; and proceed in a manner analogous to the simpler calculation discussed at p. 193. The columns for stature and cubit will be nearly the same as those of Table II. in that passage; but not quite the same. For negative values are admissible there for the deviation of stature which is there the dependent variable, but not here, where both stature and knee-height are treated as independent variables. On the other hand, negative deviations of cubit are admissible here, but not there. The rationale of this distinction is sufficiently explained by the remark at p. 194 (top).

Here is a specimen of the calculation, the columns for 
cubit and stature being here, as for the most part they must be, identical with those used for the simpler calculation (at p. 193).

TABLe III.

\begin{tabular}{|c|c||c|}
\hline $\begin{array}{c}\text { Height of knee } \\
\text { (above 20.5). }\end{array}$ & $\begin{array}{c}\text { Statures. } \\
\text { (above 67.2). }\end{array}$ & $\begin{array}{c}\text { Corresponding } \\
\text { cubit. }\end{array}$ \\
\hline $22 \cdot 9$ & $72 \cdot 4$ & $19 \cdot 3$ \\
$21 \cdot 4$ & $71 \cdot 9$ & $18 \cdot 7$ \\
$21 \cdot 6$ & $71 \cdot 5$ & $18 \cdot 8$ \\
$21 \cdot 5$ & $18 \cdot 4$ \\
$21 \cdot 7$ & 70 & $18 \cdot 6$ \\
22 & $70 \cdot 3$ & $19 \cdot 6$ \\
$21 \cdot 1$ & $62 \cdot 2$ & $18 \cdot 4$ \\
$20 \cdot 6$ & 68 & $18 \cdot 3$ \\
$21 \cdot 6$ & $71 \cdot 6$ & $19 \cdot 3$ \\
$21 \cdot 4$ & $68 \cdot 4$ & $18 \cdot 2$ \\
$20 \cdot 6$ & $68 \cdot 3$ & $18 \cdot 1$ \\
$20 \cdot 8$ & $68 \cdot 4$ & $18 \cdot 7$ \\
21 & $70 \cdot 2$ & 18 \\
$22 \cdot 3$ & $71 \cdot 1$ & $19 \cdot 4$ \\
$21 \cdot 4$ & $68 \cdot 3$ & $18 \cdot 8$ \\
\hline $321 \cdot 4$ & $1051 \cdot 6$ & $280 \cdot 6$ \\
\hline
\end{tabular}

The mean deviations of cubit and of stature are as before (in units of the quartiles) $1 \cdot 17$ and $1 \cdot 66$. The mean deviation of knee-height is $\left(\frac{321.4}{15}-20.5\right) \div \cdot 8(20.5 \mathrm{in}$. being the mean and 8 in. the quartile for the height of knee; Galton, Proc. Roy. Soc. 1888, Co-relations, Table I.) $=1 \cdot 16$. According to theory the sum of the mean positive deviations of the stature and knee-height divided into the corresponding mean deviation of cubit should yield a quotient $\cdot 42$. In fact

$$
1 \cdot 17 \div(1 \cdot 16+1 \cdot 66)=\cdot 415 \text {. }
$$

This consilience between fact and theory will appear all the more striking when it is mentioned that the anthropometrical observations from which $\mathrm{Mr}$. Galton educed his coefficients $\cdot 8, \cdot 9$, and $\cdot 8$ were not coincident with those by which I have verified the theory built upon those coefficients.

This theory is readily extended to the case of many variables. One way of looking at the whole matter is as follows:Beginning with the case of three variables-the familiar $x, y, z$-let us suppose that $w$, the probability of particular values of $x, y, z$ concurring, $=\mathrm{J} e^{-\mathrm{k}} d x d y d z$, where $\mathrm{R}=a x^{2}$ $+b y^{2}+c z^{2}+2 f y z+2 g x z+2 h x y$; and let us inquire how, 
given the coefficients in this expression, we could deduce the coefficient of correlation between any two of the variables, $x$ and $y$. The sought coefficient, say $r$, is such that to any assigned value of $x$, e. g. $x^{\prime}$, there corresponds $r^{\prime} x^{\prime}$ as the most probable value of $y$ : that is to say, the $y$ which in the long run-the long run whose stages are different values of $z$-most frequently occurs. The direct method is to substitute $x^{\prime}$ for $x$ in $w$, integrate between extreme limits with regard to $z$, differentiate with regard to $y$, and equate to zero. Geometrically we may imagine a surface in the fourth dimension of space at a distance $w_{1}$ from the plane $x=x^{\prime}$; where $w_{1}$ is what $w$ becomes when $x^{\prime}$ is substituted for $x$. The annexed diagram is intended to assist the imagination by representing the curves of probability projected on the plane $y z$. The point $\mathrm{O}_{2}$ is the centre of that system of ellipses which is formed by

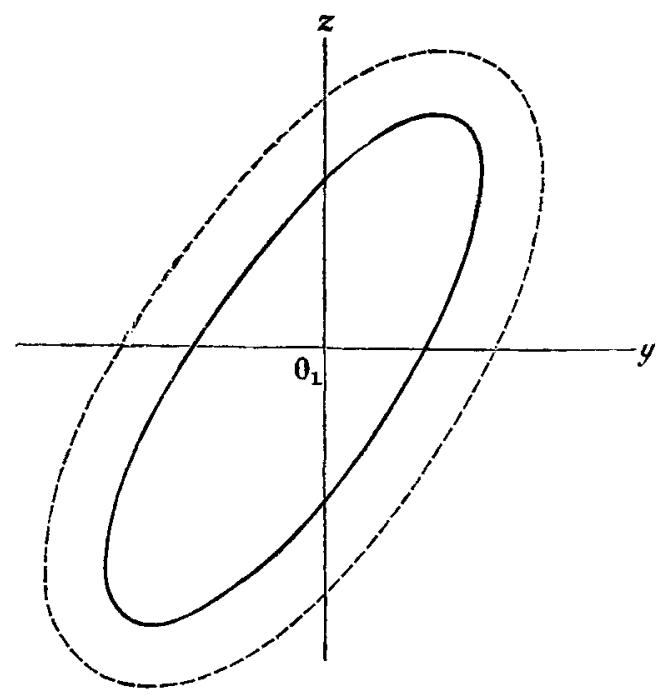

the section of the plane $x=x^{\prime}$ with the ellipsoid

$$
a x^{2}+b y^{2}+c z^{2}+2 f y z+2 g . x z+2 h x y=\text { const. }
$$

The surface $v_{1}$ is evidently symmetrical on either side of planes parallel to the axes $y$ and $z$ through the point $\mathrm{O}_{1}$. Thus the sought $y$ to which corresponds the greatest number of instances in the long run, the $y$ for which the strip $\int_{-\infty}^{+\infty} w_{1} d z$ is greatest, is the ordinate of $O_{1}$; which is also 
the $y$ for which the corresponding w-ordinate is greatest. It is given therefore by the equations

$$
\frac{d w_{1}}{d y}=0, \frac{d w_{1}}{d z}=0
$$

$w_{1}$ being what $w$ becomes when $x^{\prime}$ is substituted for $x$ ) ; or by the equations

that is

$$
\frac{d \mathrm{R}_{1}}{d y}=0, \frac{d \mathrm{R}_{1}}{d z}=0
$$

$$
\begin{aligned}
& h x^{\prime}+b y+f z=0, \\
& g x^{\prime}+f y+c z=0 .
\end{aligned}
$$

Whence

$$
y=\frac{f g-c h}{b c-f^{2}} x^{\prime}=\frac{\mathrm{H}}{\mathrm{A}} x^{\prime},
$$

where $\mathrm{H}$ and $\mathrm{A}$ are minors of the discriminant

$$
\left|\begin{array}{lll}
a & h & g \\
h & b & f \\
g & f & c
\end{array}\right|
$$

Thus $r=\frac{\mathrm{H}}{\mathrm{A}}$ as before.

By parity of reasoning the most probable $x$ corresponding to an assigned $y^{\prime}$ is $\frac{\mathrm{H}}{\mathrm{B}}$. Thus $\mathrm{A}=\mathrm{B}$. And by parity the coefficients of correlation between the other pairs $x z$ and $y z$ are respectively proportional to $\mathrm{G}$ and $\mathrm{F}$; and $\mathrm{C}=\mathrm{B}=\mathrm{A}$.

From the same point of view it appears that the coefficient of $x^{2}$ in the exponent of the expression $\iint w d y d z$ is $\frac{\Delta}{\mathrm{A}}$. For suppose for a moment that there were added to $w$ a term $-2 \mathbf{x} x$; the centre of the surface being thus tranferred from zero to $\mathrm{x}$. The most probable $x$ is found by solving the system

$$
\begin{aligned}
& a x+h y+g z=\mathbf{x}, \\
& h x+b y+f z=0, \\
& g x+f y+c z=0 .
\end{aligned}
$$

Solving for $x$, we have $\mathrm{A} x=\Delta \mathrm{x}, x=\frac{\Delta}{\mathrm{A}} \mathrm{x}$; and accordingly $\iint w d y d z$ is of the form $J e^{-\frac{\Delta}{\Lambda}(x-\mathbf{x})^{2}}$. 
But, since by convention the modulus of the variable is unity, $\Delta=\mathrm{A}(=\mathrm{B}=\mathrm{C})$; a result which continues to hold as $\mathrm{x}$ is supposed to vanish.

This reasoning is quite general; and accordingly, replacing the symbols $x_{1}, x_{2}, x_{3}$, we may extend to four and higher numbers of variables the solution which has been given above for the case of three variables. In the case of four variables $\rho_{12}, \rho_{13}, \& c . \rho_{24} \ldots$ being as before the coefficients of correlation for each pair, the reciprocal of the discriminant

$$
\Delta^{\prime}=\left|\begin{array}{llll}
\Delta, & \Delta \rho_{12}, & \Delta \rho_{13}, & \Delta \rho_{14} \cdot \\
\Delta \rho_{12}, & \Delta, & \Delta \rho_{23}, & \Delta \rho_{24} . \\
\Delta \rho_{13}, & \Delta \rho_{23}, & \Delta, & \Delta \rho_{34} . \\
\Delta \rho_{14}, & \Delta \rho_{24}, & \Delta \rho_{34}, & \Delta .
\end{array}\right|
$$

We have merely to border with a new row and column the determinant used for the case of three rariables. But in forming the first minors of the reciprocal in the case of four (and similarly for any even number) it must be observed that, according to the rule of signs*, the minor of the reciprocal which is equated to $\Delta^{2} q_{12}$ (in general $\Delta^{n-2} q_{12}$ ) is not now $\Delta^{3}\left(\rho_{23} \rho_{34} \rho_{14}\right)$, but $-\Delta^{3}\left(\rho_{23} \rho_{34} \rho_{14}\right)$; or, as it might be more elegant to write, $-\Delta^{3}\left(\rho_{23} \rho_{34} \rho_{41}\right)$. By the same rule,

$$
\begin{aligned}
& \Delta \rho_{13}=+\Delta^{3}\left(\rho_{24} \rho_{31} \rho_{42}\right), \\
& \Delta \rho_{14}=-\Delta^{3}\left(\rho_{21} \rho_{32} \rho_{43}\right),
\end{aligned}
$$

Example.-To exhibit the correlation between four quantities, of which the first, $x_{1}$, is formed by taking at random the sum of ten digits (say from a page of mathematical tables); the second, $x_{2}$, is formed by adding to the first $x_{1}$ another random decade; $x_{3}=a_{2}+$ another random decade; $x_{4}=x_{3}+$ another random decade.

The coefficients of correlation between the pairs, which have usually to be ascertained by observation, are here deducible à priori. E. $g \cdot \rho_{12}=\sqrt{\frac{1}{2}}$. For, putting $\xi_{1}$ and $\xi_{2}$ for the actual deviations of the first and second quantities (the sum of ten and the sum of twenty digits), we may regard $\xi_{1}$ and $\left(\xi_{2}-\xi_{1}\right)$ as fluctuating independently, according to a modulus which is the same for both, say $c$; being that which apper-

* Cf. Salmon's 'Higher Algebra,' chap. 1, arts. 6 and 8. 
tains to a sum of ten random digits (it is $\sqrt{165}$ ). Accordingly the equiprobable curves are

$$
\frac{\xi_{1}}{c^{2}}+\frac{\left(\xi_{1}-\xi_{2}\right)^{2}}{c^{2}}=\text { const. } ;
$$

or

$$
\frac{2 \xi_{1}^{2}-2 \xi_{1} \xi_{2}+\xi_{2}^{2}}{c^{2}}=\text { const. }
$$

Reducing to units of modulus by putting

$$
x_{1}=\frac{\xi_{1}}{c}, \quad x_{2}=\frac{\xi_{2}}{\sqrt{2} c},
$$

we have

$$
2 x_{1}^{2}-2 \sqrt{2} x_{1} x_{2}+2 x_{2}
$$

whence $\rho_{12}=\frac{1}{\sqrt{2}}$. In like manner it can be shown that

$$
\rho_{13}=\sqrt{\overline{1}}, \rho_{14}=\sqrt{\overline{1}}, \rho_{23}=\sqrt{\overline{3}}, \rho_{24}=\sqrt{\frac{\overline{2}}{4}}, \rho_{34}=\sqrt{\frac{\overline{3}}{4}} .
$$

Thus, for the determinant which is the reciprocal of the discriminant, we have

$$
\begin{aligned}
& \Delta^{\prime}=\mid \begin{array}{ccccc}
\Delta, & \Delta \sqrt{\overline{1}}, & \Delta \sqrt{\overline{1}}, & \Delta \sqrt{\overline{1}} \\
\Delta \sqrt{\overline{4}}, & \Delta, & \Delta \sqrt{\overline{2}}, & \Delta \sqrt{\overline{3}}
\end{array} \\
& \Delta \sqrt{\overline{3}}, \quad \Delta V_{\overline{3}}^{\overline{2}}, \quad \Delta, \quad \Delta \sqrt{\overline{3}}_{\overline{4}}^{\overline{3}} \\
& \Delta \sqrt{\overline{1}}, \quad \Delta \sqrt{\frac{2}{4}}, \quad \Delta \sqrt{\overline{3}}, \quad \Delta \\
& p_{11}=\Delta\left|\begin{array}{ccc}
1, & \sqrt{\overline{3}}, & \sqrt{\frac{\overline{2}}{4}} \\
\sqrt{\frac{2}{3}}, & 1, & \sqrt{\frac{3}{4}} \\
\sqrt{\frac{2}{4}}, & \sqrt{\frac{3}{4}}, & 1
\end{array}\right|=\Delta \frac{1}{1 \overline{2}},
\end{aligned}
$$




$$
\begin{aligned}
& \begin{array}{ccc}
\sqrt{\overline{3}}, & \sqrt{\frac{2}{4}}, & \sqrt{\frac{1}{2}} \\
1, & \sqrt{\overline{3}}, & \sqrt{\frac{1}{3}} \\
\sqrt{\frac{\overline{3}}{4}}, & 1, & \sqrt{\frac{1}{4}}
\end{array} \mid=-\Delta \frac{1}{12} \frac{1}{\sqrt{2}} . \\
& p_{13}=+\Delta\left|\begin{array}{rrr}
\sqrt{\frac{2}{4}}, & \sqrt{\frac{1}{2}}, & 1 \\
\sqrt{\overline{3}}, & \sqrt{\frac{1}{3}}, & \sqrt{\overline{3}} \\
1, & \sqrt{\frac{1}{4}}, & \sqrt{\frac{\overline{2}}{4}}
\end{array}\right|=0 . \\
& \text { \&c. } \\
& \text { \&c. }
\end{aligned}
$$

The determinant $\Delta$, thus determined in terms of itself, may be written :-

$$
\Delta=\left|\begin{array}{cccc}
\frac{1}{12} \Delta, & -\frac{1}{12}-\frac{1}{\sqrt{2}} \Delta, & 0, & 0 \\
-\frac{1}{12} \frac{1}{\sqrt{2}} \Delta, & \frac{1}{6} \Delta, & -\frac{1}{4} \frac{1}{\sqrt{6}} \Delta, & 0 \\
0, & -\frac{1}{4} \frac{1}{\sqrt{6}} \Delta, & \frac{1}{4} \Delta, & -\frac{1}{4} \frac{1}{\sqrt{3}} \Delta \\
0, & 0, & -\frac{1}{4} \frac{1}{\sqrt{3}} \Delta, & \frac{1}{6} \Delta .
\end{array}\right|
$$

To determine $\Delta$ we have

$$
\Delta=\Delta^{3} \times\left|\begin{array}{ccc}
\frac{1}{6}, & -\frac{1}{4} \frac{1}{\sqrt{5}}, & 0 \\
-\frac{1}{4} \frac{1}{\sqrt{ } \overrightarrow{6}}, & \frac{1}{4}, & -\frac{1}{4} \frac{1}{\sqrt{3}} \\
0, & -\frac{1}{4} \frac{1}{\sqrt{3}}, & \frac{1}{6}
\end{array}\right|=\Delta^{3} \frac{1}{576} .
$$


When $\Delta^{2}=576, \Delta=24$. Substituting the value of $\Delta$ in the expressions for the coefficients, we find for the sought quantic $R$,

$$
2 x_{1}^{2}+4 x_{2}^{2}+6 x_{3}^{2}+4 x_{4}^{2}-2 \sqrt{2} x_{1} x_{2}-2 \sqrt{6} x_{2} x_{3}-2 \sqrt{12} x_{3} x_{4} \text {. }
$$

To verify and illustrate this result, let it be employed to answer the question, What is the most probable value of $u_{1}$ corresponding to assigned values of $x_{2}, x_{3}, x_{4}$. Differentiating $\mathrm{R}$ with respect to $x_{1}$ and equating to zero, we have $2 x_{1}-\sqrt{2} x_{2}=0$; importing that to any assigned deviation of the second variable the most probable corresponding deviation of the first variable is $\sqrt{2}$ times less ; each deviation being reckoned in units of its own modulus.

The truth of this follows at once from the datum that $\rho_{12}=\frac{1}{\sqrt{2}}$. To give another proof of that proposition :-the mean positive error of the actual deviations of the first decade of digits from its mean value 45 is the modulus for such a decade, say $c, \div \sqrt{\pi}$. Now let there be added a second random decade. Its deviations being in the long run as often positive as negative, the sum of the two decades is the same as that of the first (the deviations of the first being exclusively positive). Thus to an actual mean positive deviation $\frac{c}{\sqrt{ } \pi}$ of the first quantity corresponds the same actual mean deviation $\frac{e}{\sqrt{\pi}}$ of the second quantity. But the modulus of the second quantity (the sum of two decades) is $\sqrt{2}$ times that of the first. Therefore, in units of modutus, to $x_{1}$ assigned corresponds, as the most probable value of $x_{2}$, a deviation less in the ratio $\frac{1}{\sqrt{2}}$. Wherre, by the Galtonian theorem, to $x_{2}$ assigned corresponds as the most probable value of $x_{1}$ a deviation less in the ratio $\frac{1}{\sqrt{2}}$. Which is the proposition here deduced from our formula.

The case of five variables follows in respect of the signs of the first minors the analogy of the case of three above illostrated ; the case of sis is analogous to that of four. And so on. 